\title{
The Gastric Emptying Study: Protocol Design Considerations
}

\author{
William C. Klingensmith III and Steven P. Lawrence \\ Department of Radiology, Porter Hospital, Denver, Colorado; Department of Gastroenterology, Porter Hospital, Denver, Colorado; \\ Radiology Imaging Associates, Denver, Colorado; and Arapahoe Gastroenterology, Denver, Colorado
}

\begin{abstract}
This study evaluated the intrapatient correlation for gastric emptying times with instant oatmeal and scrambled egg meals. In addition, this study evaluated the degree of overlap between the stomach and the colon or jejunum in the anterior (AP) and left anterior oblique (LAO) projections in CT studies of the abdomen. Methods: Fifteen patients were studied twice, $1 \mathrm{~d}$ apart, with instant oatmeal and scrambled egg meals, both of which were labeled with ${ }^{99 \mathrm{~m} T c}$-sulfur colloid. Imaging was performed in the upright position with the LAO projection. One-minute digital images acquired every $15 \mathrm{~min}$ were used to calculate a simple half-time of emptying. The correlation coefficient between the oatmeal and egg meals was calculated. In addition, the degree of overlap between the stomach and the colon or jejunum in the AP and LAO projections in $100 \mathrm{CT}$ studies of the abdomen was categorized as none, mild (up to 25\%), moderate $(25 \%-50 \%)$, or marked (>50\%). Results: One pair of gastric emptying studies was eliminated as an outlier because the distribution of the meals in the stomach, as determined by imaging, was very different for the 2 studies. In the remaining 14 patients, the correlation coefficient for half-times of emptying for the oatmeal and egg meals was $0.77(P<0.01)$. The stomach overlapped the colon $74 \%$ of the time in the AP projection and $82 \%$ of the time in the LAO projection ( $P=$ not significant). The stomach overlapped the jejunum $77 \%$ of the time in the AP projection but only $52 \%$ of the time in the LAO projection $(P<0.05)$. Conclusion: The significant correlation between the instant oatmeal and scrambled egg gastric emptying times suggests that similar information is provided by the 2 meals. The frequent overlap of the stomach and the colon in both the AP and the LAO projections suggests that imaging at delayed times, such as $4 \mathrm{~h}$, is likely to be problematic because of colonic activity within the stomach region of interest. In addition, the frequent overlap of the stomach and the jejunum has implications for calculation of the lag time; the less frequent overlap of the stomach and the jejunum in the LAO projection than in the AP projection may be an advantage for imaging in the LAO projection.
\end{abstract}

Key Words: gastric emptying; ${ }^{99 m}$ Tc-sulfur colloid; oatmeal; egg; delayed imaging

J Nucl Med Technol 2008; 36:195-199

DOI: $10.2967 /$ jnmt.108.054817

Received Jun. 1, 2008; revision accepted Aug. 20, 2008.

For correspondence or reprints contact: William C. Klingensmith III, $4720 \mathrm{E}$.

Oxford Ave., Englewood, CO 80113.

E-mail: bill.klingensmith@mac.com

COPYRIGHT @ 2008 by the Society of Nuclear Medicine, Inc.
$\mathbf{T}$ he gastric emptying study consists of many parameters, including patient preparation, meal composition, meal radiolabeling, patient position, imaging projection, duration of study, and data analysis. Since the gastric emptying study was first reported in 1966 by Griffith et al., numerous variations of each of these parameters have been published $(1,2)$. However, the lack of a nonradioactive reference standard makes it difficult to determine the relative merits of the many reported variations in these parameters.

In 2000, Tougas et al. reported normal values for 123 healthy subjects at 11 centers by using a standardized protocol (3). The protocol specifies a meal of scrambled egg substitute equivalent to 2 eggs, 2 slices of bread, strawberry jam, and water, with an overall caloric value of $255 \mathrm{kcal}$. The meal is labeled by cooking the egg substitute with $99 \mathrm{~m}$ Tcsulfur colloid. Patients are imaged in the upright position in the anterior and posterior projections at $0,1,2$, and $4 \mathrm{~h}$. Gastric retention of greater than the 95th percentile was set as the upper limit of normal at each imaging time, with an emphasis on the 4-h value. Because of the large number of healthy subjects in that trial, there is a movement to adopt this protocol as a recommended standard $(4,5)$.

In general, a protocol should be as simple as possible without sacrificing accuracy. We are concerned that the protocol of Tougas et al. (3) has not been compared with simpler protocols in an intrapatient fashion. In addition, we are concerned that the length of the protocol of Tougas et al., $4 \mathrm{~h}$, will allow the labeled meal to pass through the small intestine and right colon and reenter the gastric region of interest.

In the present study, we performed a direct intrapatient comparison of our standard, relatively simple gastric emptying study using a meal of instant oatmeal and our standard study using a commercially available meal of scrambled egg, both of which were labeled with ${ }^{99 \mathrm{~m}} \mathrm{Tc}$-sulfur colloid. Although we did not evaluate the more complex meal used in the protocol of Tougas et al. (3), egg is the main component in that meal and in many other previously described meals used in gastric emptying studies.

In addition, we determined the degree of overlap between the stomach and the colon as well as between the stomach and the jejunum in the anterior (AP) and left anterior oblique 
(LAO) projections in $100 \mathrm{CT}$ studies of the abdomen to assess the likelihood that gastric contents reaching the colon by 1-4 $\mathrm{h}$ would confound the results of a gastric emptying study protocol that depends on measurements from delayed images. Likewise, the frequency and degree of overlap between the stomach and the jejunum are of interest because they relate to the initial portion of the gastric emptying curve, calculation of the lag phase, and selection of the optimal imaging projection.

\section{MATERIALS AND METHODS}

\section{Patient Population}

Fifteen consecutive patients who were having a gastric emptying study for clinical reasons and who agreed to participate in the research study were enrolled. The research protocol was approved by the Institutional Review Board.

\section{Test Meals}

The standard meal for gastric emptying studies at our institution consists of one packet of instant oatmeal (Quaker Oats Co.) reconstituted with water, mixed with $18.5 \mathrm{MBq}(0.5 \mathrm{mCi})$ of ${ }^{99 \mathrm{~m}} \mathrm{Tc}$-sulfur colloid, and heated in a microwave oven. The meal has a caloric content of $100 \mathrm{kcal}$ and contains $2 \mathrm{~g}$ of fat, $4 \mathrm{~g}$ of protein, and $19 \mathrm{~g}$ of carbohydrate. In addition, one packet of natural sugar or artificial sweetener is allowed.

The comparison egg meal was obtained from a local centralized radiopharmacy (Cardinal Health) and consisted of one egg mixed with $18.5 \mathrm{MBq}(0.5 \mathrm{mCi})$ of ${ }^{99 \mathrm{~m}} \mathrm{Tc}$-sulfur colloid and scrambled in a skillet. The scrambled egg was placed in a plastic bag and transported to our institution, where it was reheated in a microwave oven.

\section{Gastric Emptying Imaging Protocol}

After overnight fasting, the patient was instructed to eat the test meal promptly. Imaging was begun when the patient finished ingesting the meal. Serial 1-min digital images were acquired every $15 \mathrm{~min}$ in the LAO projection with the patient standing. The patient was allowed to sit between image acquisitions. The routine imaging times for the oatmeal and egg meals were 60 and $90 \mathrm{~min}$, respectively. Delayed imaging was performed if the patient did not reach the half-time of emptying by the end of the routine imaging time.

\section{Analysis of Gastric Emptying Studies}

A region of interest was manually placed over the stomach on each image. The counts per region of interest on each image were corrected for radioactive decay. The data points were connected by straight lines. A simple half-time of gastric emptying was calculated. At our institution, the upper limits of normal are $60 \mathrm{~min}$ for oatmeal and $90 \mathrm{~min}$ for scrambled egg (6).

\section{CT Studies of Abdomen}

The CT studies were part of whole-body PET/CT studies or were stand-alone $\mathrm{CT}$ studies and were acquired with oral contrast material and not necessarily intravenous contrast material. Any patient with significant distortion of the abdominal contents was excluded; such distortion could be caused by, for example, a moderate to large hiatal hernia, pancreatitis, or previous upper gastrointestinal tract surgery. Accrual was continued until 100 studies suitable for evaluation were identified.
All images containing the stomach were reviewed, and the degree of overlap of the stomach and the colon or jejunum in the $\mathrm{AP}$ and LAO projections was subjectively categorized as none, mild (up to $25 \%$ ), moderate $(25 \%-50 \%)$, or marked $(>50 \%)$. Of necessity, the CT images were obtained in the supine position, although the gastric emptying studies were performed in the upright position.

\section{Statistical Analysis}

In the paired gastric emptying studies, the half-times of emptying were compared by use of the correlation coefficient. In the CT studies of the abdomen, the differences between the overlap of the stomach and the colon and the overlap of the stomach and the jejunum in the AP and LAO projections were compared by use of the $\chi^{2}$ test.

\section{RESULTS}

\section{Patient Statistics}

Among the 15 patients undergoing gastric emptying studies, there were 7 males and 8 females ranging in age from 20 to $87 \mathrm{y}$, with an average age of $52.1 \mathrm{y}$. Among the 100 patients whose CT studies of the abdomen were evaluated, there were 41 males and 59 females ranging in age from 9 to $93 \mathrm{y}$, with an average age of $50.8 \mathrm{y}$.

\section{Gastric Emptying Studies}

In all 15 patients undergoing gastric emptying studies, the instant oatmeal and scrambled egg studies were performed $1 \mathrm{~d}$ apart. The oatmeal study was performed first in 12 patients and the egg study was performed first in 3 patients. One patient was considered to be an outlier (Fig. 1, datum point at far right). In this patient, the distribution of the instant oatmeal and scrambled egg meals within the stomach was quite different; the oatmeal passed rapidly from the fundus into the body and antrum of the stomach, but a moderate amount of the egg persisted in the fundus (Fig. 2). When the outlier was excluded, the correlation coefficient in the remaining 14 patients was $0.77(P<0.01)$.

\section{CT Studies of Abdomen}

The degree of overlap of the stomach with the colon or jejunum in the AP and LAO projections is summarized in Table 1. Overlap of the stomach and colon occurred in $74 \%$ of patients in the AP projection and $82 \%$ of patients in the LAO projection; the difference was not significant. Overlap of the stomach and colon usually involved the distal transverse colon and the splenic flexure of the colon (Fig. 3). Overlap of the stomach and jejunum occurred in $77 \%$ of patients in the AP projection and 52\% of patients in the LAO projection; the difference was significant $(P<0.05)$ (Fig. 3). In general, mild overlap and moderate overlap were much more common than marked overlap for both the colon and the jejunum. A total of $92 \%$ of patients had some degree of overlap of the stomach with the colon, the jejunum, or both in both the AP and the LAO projections. 


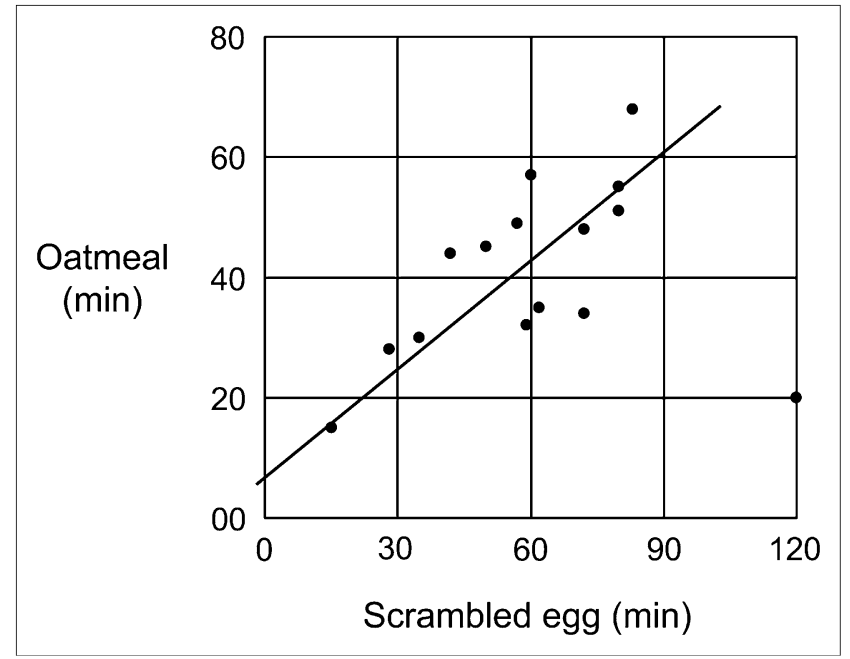

FIGURE 1. Scattergram of half-times of emptying for oatmeal

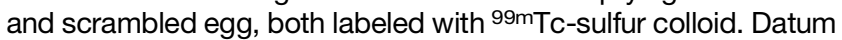
point at far right was determined to be an outlier on the basis of its location relative to those of other data points and differences in meal distribution within stomach on images. Correlation coefficient for half-times of emptying for oatmeal and scrambled egg in remaining 14 patients was $0.77(P<0.01)$.

\section{DISCUSSION}

This intrapatient comparison study revealed a relatively strong correlation between the half-time of gastric emptying of instant oatmeal and that of scrambled eggs. This correlation suggests that similar information is provided by these 2 meals. A stronger correlation between 2 different meals in a relatively small number of patients is unlikely because of the known moderate variations in emptying times in a given patient when a gastric emptying study is repeated with the same meal $(7,8)$.
Oatmeal as a test meal has the advantages of being easy to prepare and acceptable to most patients (9). In addition, oatmeal has the advantage of being semisolid, thereby minimizing the amount of time that a patient spends masticating; therefore, the meal enters the stomach in more of a bolus fashion (10). The adherence of the colloid label to the oatmeal in gastric juice in vitro has been found to be satisfactory, at $82 \%-97 \%$ (11).

For our comparison, we chose a commercially available single scrambled egg meal because it is available from a national radiopharmacy and can be replicated at other institutions. In addition, it did not require the significant on-site preparation time that more complex meals, such as the meal used by Tougas et al. (3), require. However, the time required for transportation and the necessary on-site reheating of the scrambled egg meal occasionally resulted in a test meal that was less than appetizing.

To date, there have been relatively few intrapatient comparisons of various test meals (3). In general, before a more complex meal is recommended for adoption, the meal should undergo an intrapatient comparison with a simpler meal, such as oatmeal. However, determining the accuracy of any meal and therefore its superiority is problematic because no reference standard is available. Although the results obtained with a given meal can be compared with patient symptoms, this approach is subjective and involves somewhat circular reasoning (12).

In the present study, overlap of the stomach and the colon was a frequent occurrence: $74 \%$ of the time in the AP projection and $82 \%$ in the LAO projection. In conjunction with the known normal small intestine transit time of 15 min $-5 \mathrm{~h}$, with a mean of just under $90 \mathrm{~min}$, this finding suggests that count readings from the gastric region of interest

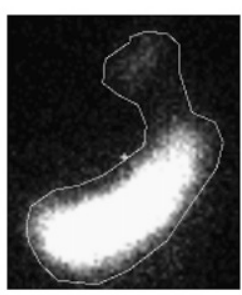

$0 \min$

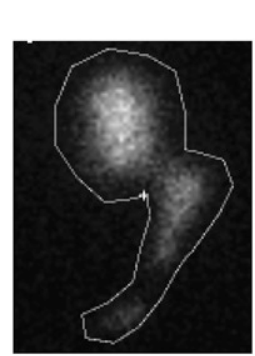

$0 \mathrm{~min}$

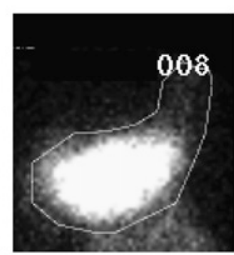

$15 \mathrm{~min}$
Oatmeal

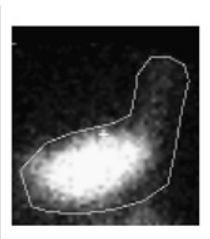

$30 \mathrm{~min}$

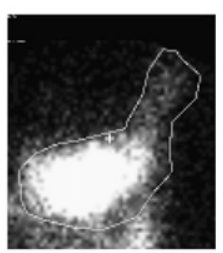

$45 \mathrm{~min}$

Scrambled Egg

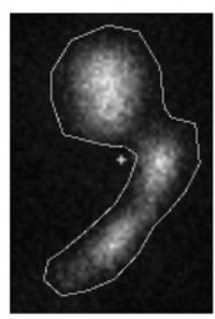

$30 \mathrm{~min}$

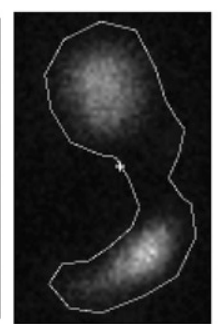

$60 \mathrm{~min}$

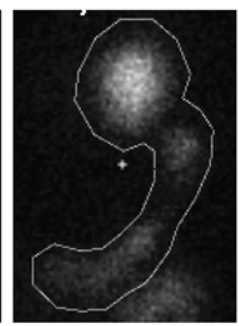

$90 \mathrm{~min}$

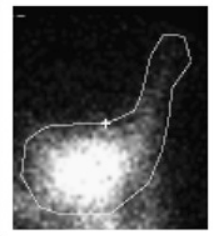

$60 \mathrm{~min}$

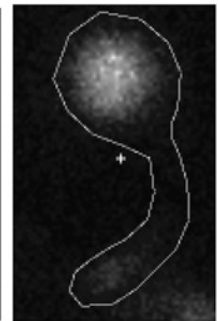

$120 \mathrm{~min}$
FIGURE 2. Images from paired oatmeal and scrambled egg studies from patient who was determined to be an outlier. Images from oatmeal study showed rapid movement of meal from fundus into body and antrum of stomach. Images from scrambled egg study showed significant retention of meal in fundus of stomach. 
TABLE 1

Overlap of Stomach and Colon or Jejunum in CT Studies of Abdomen

$\%$ of patients with indicated degree of overlap:

\begin{tabular}{ccrcccc} 
Organs & Projection & None & Mild & Moderate & Marked & Any \\
\hline $\begin{array}{c}\text { Stomach and } \\
\text { colon }\end{array}$ & AP & 26 & 51 & 20 & 3 & 74 \\
& LAO & 18 & 29 & 46 & 7 & 82 \\
$\begin{array}{c}\text { Stomach and } \\
\text { jejunum }\end{array}$ & AP & 23 & 43 & 32 & 2 & $77^{\star}$ \\
\hline $\begin{array}{c}\text { Stomach and } \\
\text { colon or }\end{array}$ & LAO & 48 & 35 & 17 & 0 & $52^{*}$ \\
jejunum & AP & 8 & & & & 92 \\
& LAO & 8 & & & & 92 \\
\hline
\end{tabular}

${ }^{\star} P<0.05$

on delayed images in a gastric emptying study will frequently include counts from the colon (13).

In the protocol of Tougas et al. (3) for gastric emptying studies, $4 \mathrm{~h}$ is recommended as the preferred measurement time for evaluating gastric emptying, with an upper limit of normal of $10 \%$ residual meal in the stomach (3). Several other authors have also recommended the use of delayed measurements, most commonly, $4 \mathrm{~h}$ (14-16). However, because counts from within the stomach are relatively low at the time of delayed imaging, a small amount of labeled meal in the overlapping colon will likely have a large impact on the counts in the gastric region of interest. Therefore, in many patients, measurement of the counts in the gastric region of interest at $4 \mathrm{~h}$ may be more indicative of the transit time from the stomach to the splenic flexure than of delayed gastric emptying.

Another finding of the present study was the frequent occurrence of overlap of the stomach and the jejunum: $77 \%$ in the AP projection and $52 \%$ in the LAO projection. This finding means that in most patients, the meal must not only pass out of the stomach but also pass through a portion of
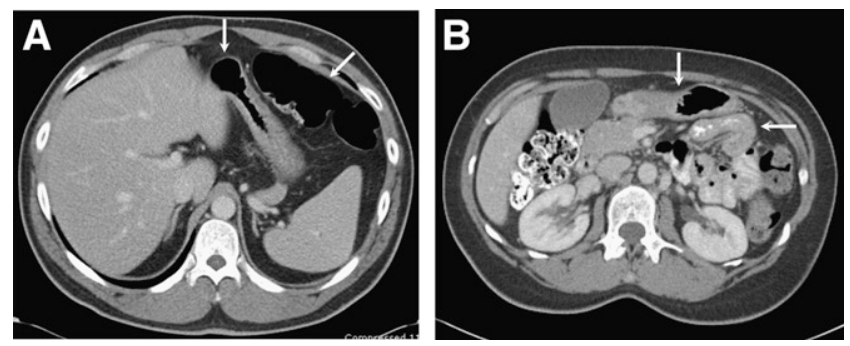

FIGURE 3. (A) CT image demonstrating overlap of stomach (vertical arrow) and splenic flexure of colon (diagonal arrow) in AP projection and, to greater extent, in LAO projection. (B) CT image demonstrating overlap of stomach (vertical arrow) and jejunum (horizontal arrow) in AP projection and, to lesser extent, in LAO projection. the jejunum before it leaves the gastric region of interest. This scenario, in turn, would generate a lag phase where none exists or would prolong a true lag phase.

The frequency of overlap of the stomach and the jejunum was significantly lower in the LAO projection than in the AP projection. This finding indicates an advantage of imaging in the LAO projection over imaging in the AP projection because there is less contamination of the gastric region of interest with jejunal activity. However, activity in the gastric region of interest can be more accurately determined in the AP projection because typically anterior and posterior images are obtained and the geometric mean method is applied to correct for attenuation (17). It is uncertain whether the AP or LAO projection is superior overall.

The problem of overlap of the stomach and the colon or jejunum cannot be solved with planar imaging. Tomographic imaging with either SPECT/CT or PET/CT would solve the problem of projectional overlap and allow precise delineation of the boundaries of the stomach. The activity in the stomach could then be segmented from that in the rest of the body for analysis. Although this approach may not be practical at present, it may become feasible as SPECT/CT and PET/CT machines become more common and less expensive.

\section{CONCLUSION}

Our findings indicate that the half-time of emptying obtained with a simple meal of instant oatmeal correlates relatively well with the results obtained with a more complex scrambled egg meal. In addition, the stomach frequently overlaps the colon, making delayed measurements of small amounts of residual meal in the stomach problematic. Finally, the stomach frequently overlaps the jejunum, although to a lesser extent in the LAO projection than in the AP projection; with implications for the choice of imaging projection and the calculation of lag time. Recommendations to standardize gastric emptying protocols with complex meals and imaging at up to $4 \mathrm{~h}$ in the AP projection are probably premature.

\section{REFERENCES}

1. Griffith GH, Owen GM, Kirkman S, Shields R. Measurement of rate of gastric emptying using chromium-51. Lancet. 1966;1:1244-1245.

2. Malmud LS, Fisher RS, Knight L, Rock E. Scintigraphic evaluation of gastric emptying. Semin Nucl Med. 1982;12:116-126.

3. Tougas G, Eaker EY, Abell TL, et al. Assessment of gastric emptying using a low fat meal: establishment of international control values. Am J Gastroenterol. 2000;95:1456-1462.

4. Abell TL, Camilleri M, Donohoe K, et al. Consensus recommendations for gastric emptying scintigraphy: a joint report of the American Neurogastroenterology and Motility Society and the Society of Nuclear Medicine. J Nucl Med Technol. 2008;36:44-54.

5. Abell TL, Camilleri M, Donohoe K, et al. Consensus recommendations for gastric emptying scintigraphy: a joint report of the American Neurogastroenterology and Motility Society and the Society of Nuclear Medicine. Am J Gastroenterol. 2008; 103:753-763.

6. Weinman J, Gritter K. Gastric emptying: normal values for a simple solid meal [abstract]. J Nucl Med. 2006;47(suppl):335P. 
7. Brophy CM, Moore JG, Christian PE, Egger MJ, Taylor AT. Variability of gastric emptying measurements in man employing standardized radiolabeled meals. Dig Dis Sci. 1986;31:799-806.

8. Lartigue S, Bizais Y, Bruley des Varannnes S, Murat A, Pouliquen B, Galmiche JP. Inter- and intrasubject variability of solid and liquid gastric emptying parameters: a scintigraphic study in healthy subjects and diabetic patients. Dig Dis Sci. 1994;39:109-115.

9. Domstad PA, Kim EE, Coupal JJ, et al. Biologic gastric emptying time in diabetic patients, using Tc-99m-labeled resin-oatmeal with and without metoclopramide. J Nucl Med. 1980;21:1098-1100.

10. Leb G, Lipp R. Criteria for labeled meals for gastric emptying studies in nuclear medicine. Eur J Nucl Med. 1993;20:185-186.

11. Fitzpatrick ML, Alderson AM. Solid food label for measurement of gastric emptying. Br J Radiol. 1979;52:920-921.
12. Guo JP, Maurer AH, Fisher RS, Parkman HP. Extending gastric emptying scintigraphy from 2 to 4 hours detects more patients with gastroparesis. Dig Dis Sci. 2001;46:24-29.

13. Kim SK. Small intestine transit time in the normal small bowel study. AJR. 1968; 104:522-524.

14. Ziessman HA, Bonta DV, Goetze S, Ravich WJ. Experience with a simplified, standardized 4-hour gastric-emptying protocol. J Nucl Med. 2007;48:568-572.

15. Thomforde GM, Camilleri M, Phillips SF, Forstrom LA. Evaluation of an inexpensive screening scintigraphic test of gastric emptying. J Nucl Med. 1995;36:93-96.

16. American Gastroenterological Association Clinical Practice Committee. Technical review on the diagnosis and treatment of gastroparesis. Gastroenterology. 2004;127:1592-1622.

17. Ford PV, Kennedy RL, Vogel JM. Comparison of left anterior oblique, anterior and geometric mean methods for determining gastric emptying times. $\mathrm{J} \mathrm{Nucl}$ Med. 1992;33:127-130. 